\title{
Como os objetos da Internet das Coisas se comportam? Uma análise através de métricas quantitativas
}

\author{
Jamisson J. Júnior ${ }^{\circledR}$, Thiago S. Figueiredo ${ }^{\circledR}$, \\ Luiz C. B. Torres, Bruno P. Santos \\ Departamento de Computação e Sistemas \\ Universidade Federal de Ouro Preto (UFOP) \\ Minas Gerais, Brasil. \\ \{jamisson.junior, thiago.figueiredo\} @aluno.ufop.edu.br \\ \{luiz.torres, bruno.ps\}@ufop.edu.br \\ ${ }^{\circledR}$ Estes autores contribuíram igualmente para este trabalho.
}

\begin{abstract}
Everyday objects have been connected promoting the Internet of Things (IoT) In this cybernetic environment, mobility is a factor that IoT's solutions must be aware. Also, IoT devices eventually will do social ties emerging the Social Internet of Things (SIoT) However, there is a lack of empiric data or even synthetic ones that allow the study of mobility patterns and interactions of IoT's entities. In this way, this paper aims to adapt an existing mobility model to mimic mobile IoT's devices and then apply metrics to quantify their interactions. Also, we run different routing protocols solutions for delayed tolerant communication over this model. The results highlights the importance of mobile objects in the IoT context and the need for deep research in this field. All data employed in this work is available online.
\end{abstract}

Resumo. Objetos do nosso cotidiano estão cada vez mais conectados formando o que se chama de Internet das Coisas (IoT). Neste ambiente cibernético conectado, a mobilidade é fator presente e soluções para IoT devem estar cientes disso. Também, os dispositivos eventualmente poderão fazer laços sociais surgindo então a Internet das Coisas Sociais (SIoT). No entanto, dados empíricos elou sintéticos que permitam o estudo do padrão de mobilidade das entidades da IoT bem como suas interações são escassos. Sendo assim, este artigo visa adaptar um modelo de mobilidade existente para mimetizar a mobilidade de dispositivos daIIoT e então aplicar métricas para quantificar as interações e os impactos no desempenho de solução de comunicação tolerantes a atraso. Os resultados aqui obtidos salientam a importância de objetos móveis no contexto do IoT Todos os dados utilizados neste artigo estão disponíveis online.

\section{Introdução}

A Internet of Things (IoT) está cada vez mais presente em nosso cotidiano, e nesta perspectiva, objetos do dia-a-dia passam a ter capacidade de sensoriamento de contexto e comunicação. Este fato potencializa as funcionalidades originais destes dispositivos e torna a computação mais ubíqua. Neste contexto, oportunidades emergem tanto do ponto de vista acadêmico quanto do industrial, possibilitando o desenvolvimento de soluções que tragam valor aos humanos [Santos et al., 2016, 2020; Kassab and Darabkh, 2020]. 
A mobilidade é fator presente em nossa rotina e dessa forma a IoT precisa de soluções computacionais flexíveis e adequadas a esta premissa. Em um cenário cibernético conectado e móvel surgem ao menos dois novos paradigmas: Internet of Mobile Things (IoMT) e Social Internet of Things (SIoT). No primeiro, os objetos possuem a liberdade de mover e as soluções devem estar cientes disso [Meslin et al., 2020]. Já no paradigma SIoT, os objetos fazem laços sociais de diferentes graus uns com os outros [Atzori et al., 2012; Marche et al., 2020].

É possível afirmar então, que na Internet do futuro a maioria das interações não serão estabelecidas entre humanos, mas entre os dispositivos considerados "inteligentes" [Castro et al., 2012]. Em outras palavras, as aplicações da IoT serão baseados nas conexões entre objetos e em muitas situações essas interações apenas serão possíveis quando os objetos estiverem próximos uns dos outros. Por exemplo, em Atzori et al. [2012], os autores fazem previsões de quais serão os tipos de relações sociais que os objetos farão do ponto de vista da SIoT. Pensando nisso, entender o movimento e interações dos objetos se torna uma necessidade para avanços na IoT e paradigmas (IoMT e SIoT).

Essa preocupação acerca do comportamento dos objetos inteligentes abrange não apenas soluções dentro da área de tecnologia. Segundo Surowiecki [2005], existem evidências científicas de que um grande número de indivíduos vinculados a uma rede social pode fornecer respostas muito mais precisas para problemas complexos do que um único indivíduo. Diante disso, entender essa nova estrutura de comunicação possibilita compreender a cultura de um determinado grupo de pessoas [Inocêncio and Ribeiro, 2020], a diversidade social [Hristova et al., 2016], o padrão de mobilidade de uma comunidade [Chang et al., 2021] e diversas outras informações que auxiliarão na tomada de decisões para a sociedade.

Portanto, este artigo visa explorar o conhecimento que se tem sobre a possível mobilidade e interações dos objetos da IoT. Duas perguntas guiam o estudo aqui proposto: i) Como estudar a mobilidade dos objetos inteligentes? ii) Como medir as interações entre objetos? Esse artigo não pretende resolver por completo essas questões, mas sim dar passos em direção às respostas. Para tanto, algumas aproximações serão propostas. Isso se deve ao fato de, no melhor de nossos conhecimentos, não existirem dados reais sobre como entidades da IoT se comportam. Dessa forma, uma adaptação ao modelo de mobilidade Small World In Motion (SWIM) [Mei and Stefa, 2009] foi proposta, pois esse modelo produz um padrão de mobilidade que se aproxima de rastros reais [Meroni et al., 2010]. Em seguida, para analisar as interações dos dispositivos, utilizamos medidas de grafos e métricas de mobilidade. Já para estudar o comportamento dos objetos em redes móveis, executamos diferentes protocolos de roteamento tolerantes a atraso e analisamos métricas tradicionais como taxa de entrega e número de transmissões. Os resultados encontrados realçam o potencial da aplicação de métricas quantitativas para análise do paradigmaSIoT, de tal forma que foi possível salientar a importância dos objetos móveis no conjunto de dados utilizado.

O restante do artigo está organizado da seguinte forma: A Seção 2 descreve os trabalhos relacionados que influenciam nosso estudo. Em seguida, na Seção 3 , a metodologia para coleta de dados é apresentada. Na Seção 4, nossa estratégia para o processamento de dados é descrita e as análises que foram abordadas são mostradas. Na Seção 5 , são detalhadas as metodologias adotadas na condução dos experimentos e os resultados 
obtidos. Finalmente, a Seção 6 traz as conclusões.

\section{Trabalhos Relacionados}

$\mathrm{Na}$ literatura existem diversos assuntos que abrangem a IoT, bem como a abordagem da mobilidade e interação dos objetos inteligentes. No artigo de Asl et al. [2013], o foco principal foi a análise das interações entre os objetos da IoT. Seu principal objetivo foi entender se, e em que medida, essas interações podem ser exploradas para configurar um grafo de objetos nos quais se possa basear algoritmos, protocolos e aplicações de qualquer SIoT futuro. Para isso, a co-presença das coisas foi um dos meios pelos quais se estabeleceu uma interação social entre os objetos. Porém poucas métricas de mobilidade foram exploradas, além disso, em sua modelagem cada pessoa recebe um conjunto de objetos baseado no tipo de lugar que ela está e não em um conjunto de dispositivos pessoais previamente estabelecidos.

Estudos voltados para a análise de redes sociais estabelecidas entre telefones celulares estão disponíveis na literatura. No trabalho de Eagle et al. [2009], os autores utilizaram para as análises um histórico de chamadas telefônicas, propondo que as relações sociais dos dispositivos eram derivadas das interações entre seus proprietários. Uma abordagem complementar foi usada nos artigos de Kostakos and Venkatanathan [2010] e Bigwood et al. [2008]. Porém nesses estudos, até onde se sabe, o único objeto em foco foi o celular, embora, na atualidade pessoas carregam consigo muitos outros objetos inteligentes como relógios/pulseiras, óculos, tablets, dentre outros. Além disso, existem outros dispositivos conectados à rede associados ao nosso cotidiano, que normalmente não se movem com o seu proprietário, e são importantes para o entendimento da dinâmica da rede, exemplo desses são os computadores, as impressoras e etc.

Em Marche et al. [2018] é proposto um conjunto de dados baseados em objetos reais da IoT, disponíveis na cidade de Santander 1 , categorizados via Modelos de Dados FIWARE ${ }^{2}$ Os dados obtidos foram disponibilizados para a comunidade, desta forma, é possível testar várias propostas em torno das comunicações objeto-a-objeto. Além disso, as simulações realizadas mostraram como regras diferentes para a criação de relacionamentos impactam a navegabilidade geral da rede. Porém, as métricas tradicionais na análise de mobilidade (por exemplo, tempo de inter-contato, raio de giro, etc.) e relações sociais não foram aplicadas para compreender o comportamento dos objetos inteligentes.

Apesar da relevância do tema e das diversas possibilidades da SIoT, esforços na criação e gestão dos enlaces sociais ainda são necessários. Nesse sentido, o artigo de Atzori et al. [2019] tem como foco o desenvolvimento de uma arquitetura para gerenciar os links sociais entre os dispositivos da rede. Entretanto, uma análise complementar sobre as entidades da SIoT contribuiria para o desenvolvimento de uma arquitetura mais robusta.

No trabalho de Aljubairy et al. [2020] foi avaliado a possibilidade de estabelecer um relacionamento entre objetos do IoT quando há co-ocorrência (tempo e espaço). Foram desenvolvidos novos algoritmos para um framework de predição de contatos, SIoT Predict. O primeiro algoritmo identifica as permanências de objetos IoT extraindo os locais correspondentes. O segundo algoritmo, denominado Sweep Line Time Overlap,

\footnotetext{
1“Tus santander,” 2021. [Online]. Disponível em: http://www.tusantander.es

2 "Fiware data models," 2021. [Online]. Disponível em: https://www.fiware.org/developers/data-models
} 
descobre quando e onde dois objetos IoT se encontraram. Após isso, foram realizados estudos para prever relacionamentos futuros, porém pouco aprofundou-se no estudo da relação objeto-objeto, de maneira que a aplicação de métricas quantitativas validaria o cenário predito.

O trabalho aqui apresentado se diferencia dos demais em ao menos um aspecto: estudar possíveis métricas que possam auxiliar no entendimento de uma rede SIoT. Para tanto, adaptamos o modelo de mobilidade sintética Small World In Motion (SWIM), de maneira similar ao apresentado por [Asl et al., 2013], de forma que cada agente simulado passou a ter objetos fixos e móveis capazes de interagir entre si e com outros da rede. Com relação às análises, levamos em consideração que esses objetos podem se mover e são capazes de formar laços sociais, dessa forma, diferentes indicadores quantitativos foram utilizados para traduzir, em números, o comportamento dos dispositivos participantes desse sistema. Por fim, observamos também que os contatos entre esses objetos possibilitam a troca de informações, logo entender a qualidade dessa transmissão é de suma importância. A seguir detalhamos os dados utilizados neste trabalho.

\section{Conjunto de dados}

No trabalho aqui apresentado utilizou-se de alguns conjuntos de dados para realização da metodologia e análises. Com o objetivo de facilitar a reprodutibilidade do artigo, juntamente em auxiliar que futuras melhorias sejam realizadas dentro da área, o conjunto de dados utilizados neste artigo, ou seja, não apenas a Tabela 11, como também o rastro de posição dos objetos gerado juntamente com seus parâmetros, estão disponíveis online ${ }^{3}$.

A par disso, a primeira fonte de dados utilizada foi obtida no relatório de dispositivos realizada pela Global Web Index ${ }^{4}$ que destaca as últimas tendências de consumo e uso das principais categorias de dispositivos. A pesquisa foi realizada em 2019 utilizando um questionário online com mais de 688 mil usuários da Internet com idade entre $16 \mathrm{e}$ 64 anos. A Tabela 1 mostra os resultados deste estudo que relaciona população e seus dispositivos. Essa informação foi utilizada para definir o conjunto de objetos que cada pessoa possui em nosso modelo.

Para adicionar mobilidade aos dispositivos em nosso modelo, geramos um rastro (trace) de locomoção sintético baseado no modelo SWIM. Para gerar esses dados utilizouse a ferramenta de geração e análise de cenários de mobilidade BonnMotion [BonnMotion, 2016], que teve como arquivo de entrada os parâmetros para simular o experimento Infocom06, disponível no site oficial do SWIM.

É importante ressaltar que os dados coletados e produzidos possuem algumas limitações, muitas delas são decorrentes da falta de dados reais dentro da literatura. A primeira delas é a falta de informação dentro do Global Web Index a cerca de usuários com múltiplos dispositivos de uma mesma categoria, como por exemplo, pessoas proprietárias de dois smartphones. Nesse sentido considerou-se que cada individuo teria apenas um único dispositivo de uma mesma categoria, baseado na Tabela 11. Além disso, em um cenário real cada dispositivo possui raio de comunicação diferente, que depende do tipo de serviço disponível, do hardware, dentre outros fatores. Para fins de simplificação,

\footnotetext{
3“Dados de entrada para a geração dos traces" 2021. [Online]. Disponível em: https://bit.ly/3jvNVTL

4 "Global Web Index". 2021. [Online]. Disponível em: https://www.globalwebindex.com/reports/device

5 "Smal World in Mortion", 2019. [Online]. Disponível em: http://swim.di.uniroma1.it
} 
Tabela 1. Porcentagem de usuários para cada dispositivo

\begin{tabular}{lcc}
\hline Dispositivo & População (\%) & Mobilidade \\
\hline Smartphone & 94 & Móvel \\
PC/Laptop & 69 & Móvel \\
Tablet & 37 & Móvel \\
Smartwatch & 13 & Móvel \\
Smart wristband & 10 & Móvel \\
VR headset & 4 & Móvel \\
Smart TV & 38 & Estático \\
Games console & 21 & Estático \\
TV streaming device & 14 & Estático \\
Smart home product & 11 & Estático \\
\hline
\end{tabular}

adotou-se um raio de comunicação de de 100 metros para todos os dispositivos [Tjensvold, 2007].

Por fim, muito dos resultados aqui encontrados tiveram influência do modelo de locomoção sintético SWIM, que mesmo se aproximando do real, possui limitações que podem afetar os resultados. Vale ressaltar que esse padrão é amplamente utilizado dentro da simulação de locomoção, logo, as métricas aqui encontradas puderam evidenciar as tendencias existente dentro de traces baseados nesse modelo.

\section{Materiais e métodos}

Após coleta dos dados iniciou-se a etapa de manipulação das informações. Esse estágio foi dividido nas partes de pré-processamento dos dados e nas análises sociais e de mobilidade. Com isso, pretendeu-se entender como os dispositivos se relacionam e descrever, por meio de algumas métricas, o comportamento desses objetos.

\subsection{Pré-processamento}

Os rastros de mobilidade trazem informações sobre como entidades se locomovem em uma determinada área. A etapa de pré-processamento se deu em eliminar informações não importantes dentro do nosso contexto e formatar os dados de modo a obter informações relevantes. Com isso em mente, adaptamos os rastros de mobilidade humana, gerados pelo SWIM, e os transformamos em um conjunto de dados que descreve como os dispositivos que esses humanos carregam se movimentam. Ademais, foi necessário identificar onde e por quanto tempo ocorreu contatos entre os objetos, levando em consideração quando dois objetos estão dentro do raio de comunicação um do outro.

Para isso, definimos um conjunto de objetos entre fixos e móveis, os quais são associados a uma pessoa. A construção desse conjunto usou como base a distribuição apresentada na Tabela 11. Foi definido que os objetos móveis seguem as trajetórias do indivíduo, já os objetos fixos são alocados na posição em que o seu proprietário está no início da simulação (posição conhecida como home location) e permanecem até o final do ensaio. Essa proposta condiz com o modelo de locomoção utilizado, uma vez que o SWIM determina que a home location do nó é a sua posição inicial, em outras palavras, 
Tabela 2. Registro de encontros entre dispositivos

\begin{tabular}{ccccc}
\hline $\mathbf{U}$ & $\mathbf{V}$ & Inicio (s) & Fim (s) & Overlapped (s) \\
\hline 45-Tablet & 54-Smartwatch & 9715.0 & 9906.8 & 191.8 \\
16-Smartphone & 36-Smartphone & 130731.2 & 131214.1 & 482.9 \\
32-Smartphone & 51-Tablet & 206248.9 & 206804.1 & 555.2 \\
75-Smartphone & 9-PC/Laptop & 60916.2 & 61763.0 & 846.8 \\
59-TV StreamingDevice & 226-Smartwristband & 162218.6 & 162312.6 & 94.0 \\
\hline
\end{tabular}

os objetos fixos ficam posicionados na casa de seu proprietário e os objetos móveis são carregados pelo mesmo para todos os locais que ele visita

Após a transformação de um rastro de posição baseada em "humano" para um rastro de objetos, a etapa de identificar os encontros e o seu tempo de duração é executada. Para esse fim, foi associado para cada objeto um raio de comunicação de 100 metros, de forma que um encontro ocorreria se dois objetos estivessem próximos o bastante ao ponto de entrarem no raio de comunicação um do outro. Para cada encontro foi registrado o tempo de início e fim de comunicação e a posição final dos nós um instante antes da comunicação acabar. Na Tabela 2 é possível visualizar uma amostra de como os encontros foram registrados, de forma que $U$ e $V$ são os identificadores das pessoas e a categoria do dispositivo que eles estavam carregando, além disso, Início e Fim indicam o tempo em segundos que o encontro foi realizado. É importante ressaltar que para não interferir nos cálculos das métricas retiramos todos os contatos entre os objetos do mesmo dono, isso porque esses objetos, sobretudo os móveis, sempre estão conectados uns com os outros e não trouxeram informações relevantes sobre o comportamento da rede.

A partir do conjunto de dados dos encontros foi possível modelar uma rede social de dispositivos utilizando um grafo. Nessa estrutura, os nós representam os dispositivos e as arestas indicaram se ocorreu contato entre os mesmos. O peso de cada aresta foi dado pelo tempo que os dois nós ficaram conectados durante toda a simulação. Visamos com essa estrutura entender qual a importância social de cada dispositivo e visualmente identificar como a rede estava se comportando.

Seguindo a mesma abordagem da Seção 3, os rastros gerados após o processamento também foram disponibilizados de forma online ${ }^{7}$. Além das infomações demostradas na Tabela 2, o conjunto de dados disponibilizado contem ainda informações do tempo de contato (overlapped) e das posições cartesianas dos nós $U$ e $V$. Os próximos tópicos descrevem as análises extraídas a partir destes dados.

\subsection{Análises}

Para a primeira análise utilizou-se o grafo de contatos e com isso foi possível extrair informações relevantes, sobretudo no quesito da importância dos nós dentro da rede. Para isso, utilizou-se as centralidades degree, eigenvector, closeness, betweenness e pagehank, como definido em Zinoviev [2018]. Já a segunda etapa se deu em aplicar métricas de mobilidade dentro do trace de contato dos dispositivos. Nesta fase foi empregado o MObility CHaracterization Framework (MOCHA) [de Souza et al., 2018], um framework para analisar traces reais e sintéticos de forma quantitativa e com métricas específicas classificadas

\footnotetext{
${ }^{6}$ Note que esse procedimento gera apenas uma caricatura do que realmente aconteceria.

7 “Dados gerados após o processamento" 2021. [Online]. Disponível em: https://bit.ly/3husWhi
} 
como: social, temporal ou espacial. Normalmente, não há padronização dessas métricas nos demais trabalhos, daí surge a importância desse framework. A seguir descrevemos quais métricas de mobilidade foram empregadas.

\subsubsection{Métricas de mobilidade}

A análise quantitativa é importante no estudo de traces pois permite testar e validar aplicações a serem usadas em cenários reais. Sendo assim, o MOCHA fornece dados importantes para nosso estudo, ampliando o entendimento do comportamento dos objetos da IoT. Uma breve descrição sobre as principais métricas será abordada a seguir, para mais detalhes o leitor pode consultar o trabalho de Souza et al. [2018].

- Inter Contact Time (INCO) - Esta métrica é definida como o intervalo de tempo entre encontros consecutivos de um par de nós. Ou seja, é possível analisar quanto tempo dois objetos ficam sem conexão entre si.

- Contact Duration (CODU) - Como o nome sugere, essa métrica indica o tempo de duração do contato entre um par de nós. Sendo útil para, por exemplo, o levantamento da quantidade de dados que podem ser transmitidos.

- Maximum Contact (MAXCON) - Esta métrica permite entender a quantidade de contatos que um objeto realiza em um determinado período de tempo. Isso é interessante pois permite definir qual é o melhor momento para troca de dados.

- Encounter Regularity (EDGEP) - Quantifica a persistência de encontros entre dois nós. Desta forma é possível inferir se há uma relação social forte, isto é, objetos que tendem a se encontrar de forma regular.

- Topological Overlap (TOPO) - Avalia a similaridade entre os nós utilizando como base os contatos em comum. Sendo assim, nós que apresentam alto valor de TOPO são mais prováveis de pertencer a um mesmo grupo, e por isso, ter mais "amigos" em comum.

- Radius of Gyration (RADG) - Esta métrica indica a distância que um dispositivo orbita em torno da sua casa. Em análises de mobilidade é comum atribuir aos nós um local do tipo casa, e no framework utilizado, o local mais visitado por um objeto é considerado como sua casa. Esta métrica pode ser útil para estudar o quão longe os dispositivos viajam em relação a sua casa.

- Travel Distance (TRVD) - É definido como a distância entre dois lugares consecutivos visitados por um nó. Este tipo de análise permite entender melhor como um nó se move de um lugar para outro, podendo ser aproveitada para melhorar a disseminação de dados em redes oportunísticas e veiculares.

- Travel Time (TRVT) - É análogo ao travel distance, porém neste caso analisa-se o tempo despendido na viagem de um local para outro.

- Contact Entropy (CONEN) - Esta métrica calcula a diversidade de dispositivos encontrados por um determinado objeto. Desta forma, um nó terá alto CONEN caso encontre com muitos outros dispositivos distintos e baixo caso contrário.

- Spatial Variability (SPAV) - Similar ao CONEN, porém aqui a métrica quantifica a diversidade de locais visitados por um nó. Dessa forma, o objeto terá baixo SPAV se a distribuição das visitas estiver concentrada em poucos locais. 


\subsubsection{Comunicação em rede}

Além de extrair métricas dos modelos de mobilidade sintéticos, é possível também utilizalos na avaliação de protocolos de rede móveis. A ideia é que esses modelos possam mimetizar o comportamento das entidades móveis e, portanto, como os protocolos reagiriam em um cenário do mundo real. A seguir, será apresentado alguns protocolos de encaminhamento de redes oportunísticas usando nossa adaptação para indicar o comportamento de entidades móveis da IoT. Avaliamos o desempenho de 4 estratégias de encaminhamento implementadas no The One Simulator [Keränen et al., 2009] descritas brevemente a seguir.

- Epidemic: funciona através do encaminhamento das mensagens sempre que um nó encontra com outro nó que não possui a mensagem ainda. Por utilizar um mecanismo similar à inundação, esta abordagem em cenários de grande escala se torna custoso devido a quantidade de retransmissões necessárias, entretanto podemos interpretar essa abordagem como um baseline para um limiar superior para entrega de mensagens bem como custo para entrega (overhead).

- First Contact: essa simples estratégia encaminha uma única cópia das mensagens no primeiro contato possível (independente do destinatário da mensagem).

- Direct Delivery: nessa estratégia as mensagens serão entregues somente no encontro com o destinatário final da rede. Sendo portanto, uma estratégia econômica do ponto de vista de overhead na rede em prejuízo da rapidez na entrega das mensagens (note que esta rapidez possui associação como a mobilidade dos nós na rede, por exemplo, se a rede possui altos valores de, por exemplo, RADG, CONEN $\mathrm{e}$ SPAV] o desempenho deste protocolo pode ser melhorado).

- PRoPHET: assume que a maioria das entidades móveis não possuem movimento completamente aleatório. Em outras palavras, seus movimentos podem ser preditos (ex.: se um local foi visitado no passado é possível que este local será visitado no futuro) [Lindgren et al., 2003]. Baseando-se nisso, PRoPHET usa uma métrica chamada delivery predictability de cada nó para cada destinatário na rede. Esta métrica indica a chance que um nó tem de entregar uma mensagem para um destinatário. Quando um nó encontra com outro essa informação é trocada e atualizada entre eles. Essa informação é utilizada no processo de escolha de encaminhamento das mensagens.

O tópico a seguir levanta os resultados e as análises obtidas a partir da aplicação dessas métricas dentro dos traces gerados.

\section{Resultados}

Para ter uma estimativa numérica, utilizamos métricas de redes sociais. As centralidades, por exemplo, são aspectos individuais de cada nó e para analisar por categoria de dispositivos optou-se por somar os valores da centralidade, o que resultou na Tabela 3 . Os dispositivos móveis foram os objetos com maior centralidade dentro da rede na maioria das métricas, sobretudo os smartphones que obtiveram destaque em todos os aspectos. Em outras palavras, dentro do nosso rastro de posições, os objetos móveis não apenas foram aqueles que realizaram mais enlaces, como também possuíram vizinhos de importância relevante dentro do modelo aplicado (como pode ser visto nas colunas degree e 
eigenvector). Somado a isso, esses objetos também serviram como pontes de conexão entre clusters e possuem distância mais curta com os demais nós da rede. Em geral, a mobilidade dos dispositivos é importante para disseminação de informação. Vale ressaltar ainda que essa análise condiz com os estudo apresentados em outros artigos [Asl et al., 2013].

Tabela 3. Valores das centralidades para cada grupo de dispositivos

\begin{tabular}{lccccc}
\hline & Degree & Closeness & Betweenness & Eigenvector & Pagerank \\
\hline Smartphone & 66.04 & 66.56 & 0.08 & 5.07 & 0.34 \\
PC/Laptop & 48.56 & 48.95 & 0.05 & 3.73 & 0.25 \\
Tablet & 26.34 & 26.59 & 0.03 & 2.02 & 0.13 \\
Smart TV & 15.71 & 20.08 & 0.00 & 1.29 & 0.09 \\
Smartwatch & 9.17 & 9.23 & 0.01 & 0.70 & 0.05 \\
Games console & 9.07 & 10.81 & 0.00 & 0.75 & 0.05 \\
Smart wristband & 7.22 & 7.29 & 0.01 & 0.56 & 0.04 \\
TV streaming device & 5.24 & 6.86 & 0.00 & 0.43 & 0.03 \\
Smart home product & 3.99 & 5.41 & 0.00 & 0.33 & 0.02 \\
VR headset/device & 2.78 & 2.80 & 0.00 & 0.21 & 0.01 \\
\hline
\end{tabular}

A Figura 1 apresenta o grafo resultante, tal que na Figura 1(a) não se aplica filtro. Para melhorar a visualização reduziu-se a amostra contendo apenas os nós cujo a soma do tempo de suas conexões era maior que $12 \mathrm{~h}$ e que também realizaram mais do que sete conexões (valores escolhidos apenas para demonstração). Dessa forma, na Figura 1) (b) é possível visualizar os elementos de destaque do ensaio, onde, os nós com maior tamanho são aqueles que possuem alto grau de conexões, já aqueles com tons mais vermelhos se referem aos nós com maior centralidade betweenness.

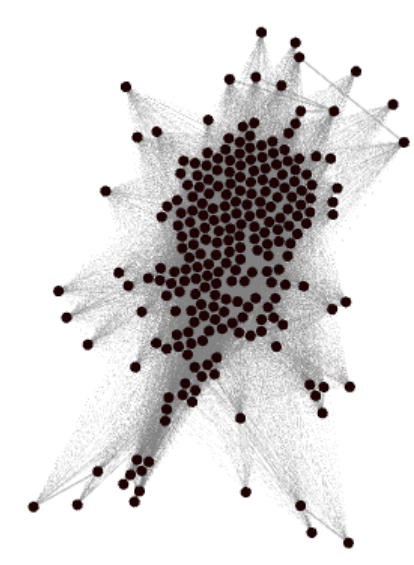

(a)

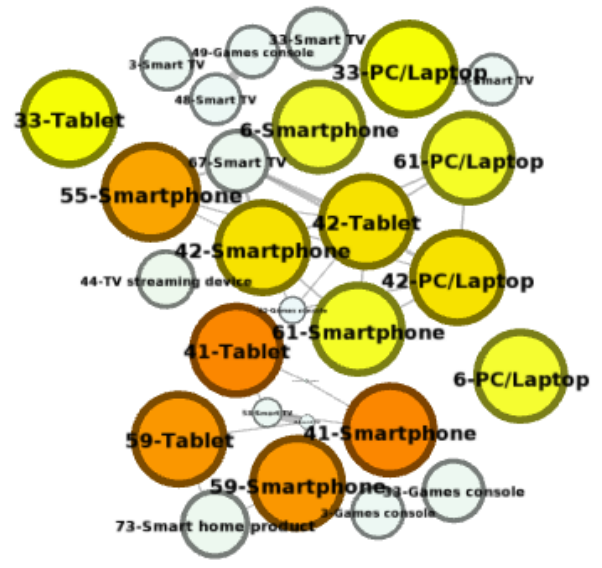

(b)

Figura 1. Grafo completo das conexões (a) e filtragem realçando dispositivos de destaque (b)

Já no quesito mobilidade, a duração e o número de contatos entre os dispositivos foram contabilizadas. A Figura 2 (a) apresenta a densidade de probabilidade do tempo 
em que os objetos estiveram em contato. Esses dados seguem uma função power law, o que indica que a maioria dos dispositivos ficaram conectados por pouco tempo. Outra informação que também segue um comportamento semelhante foi a função de densidade do número de contatos, nesse caso, a Figura 2(b) indica que é mais comum que haja poucos contatos entre um mesmo par de objetos. Sendo assim, é possível inferir que a maioria dos pares não possui relação social forte entre si, o que é característica de uma rede social complexa [Zinoviev, 2018].

Ampliando a análise do tempo de contato, separamos a densidade de probabilidade entre objetos móveis e objetos fixos de acordo com o CODU médio na Figura 2(c). A partir disso, percebe-se que os objetos fixos tendem a realizar contatos mais duradouros, ou seja, conseguem transmitir mais dados entre si e com seus donos. Portanto, em cenários oportunísticos, os dispositivos fixos podem ser utilizados como pontos estratégicos para disseminação de informações entre os objetos que eventualmente o encontram.

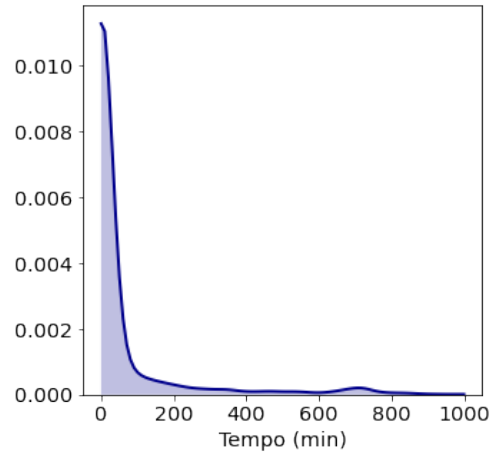

(a)

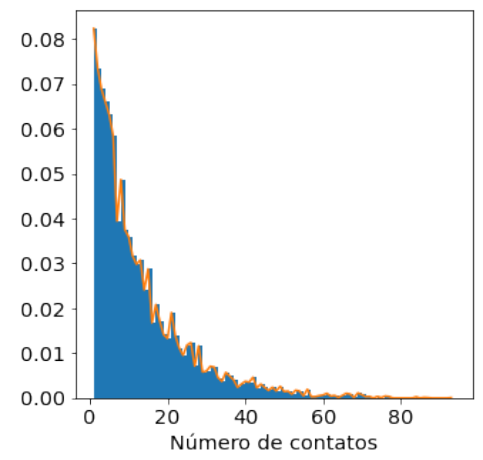

(b)

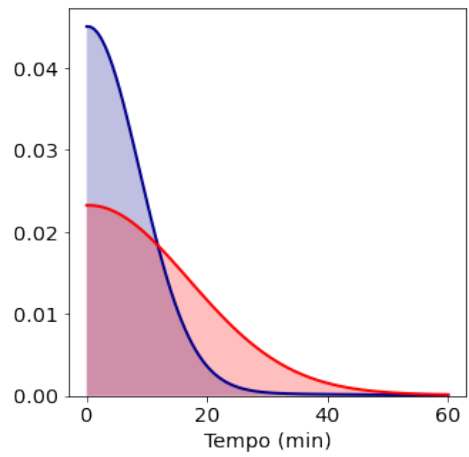

(c)

Figura 2. Densidade de probabilidade do (a) tempo de contato entre pares de objetos, (b) Número de contatos dos dispositivos, e (c) CODU médio de objetos fixos (vermelho) e móveis (azul)

Em seguida, analisou-se o EDGEP, ou seja, a frequência de encontros entre os pares de objetos. A densidade de probabilidade mostrada na Figura 3 (a) permite visualizar que encontros frequentes são mais raros. Informação esta que reafirma o que foi apontado anteriormente, ou seja, poucos objetos possuem relação social forte. Ainda sobre a regularidade de encontros, objetos estáticos possuem menor valor quando comparado à objetos que se movem. O que faz sentido, pois objetos fixos só encontram outro dispositivo quando este passar por ele novamente. Portanto, quando se tratando de cenários oportunísticos, objetos móveis são capazes de trocar dados com maior frequência.

Analisando o TOPO, isto é, contatos em comum entre objetos, observa-se que dispositivos que se movem tem muitos contatos em comum quando comparados aos objetos estáticos, conforme visto na Figura 3(b). Desse modo, os objetos que se movem são mais importantes na troca de dados entre membros de um mesmo grupo. Já a análise do tempo médio entre contatos, INCO, permite concluir que os nós que se movem tendem a ficar menos tempo entre contatos. O que é esperado visto que dispositivos móveis possuem mais chances de se conectar com algum nó pois o número de contatos por hora (MAXCON destes é maior conforme mostra a Tabela 4

Uma análise conjunta da distância viajada, TRVD, e do raio de giro, RADG, mos- 


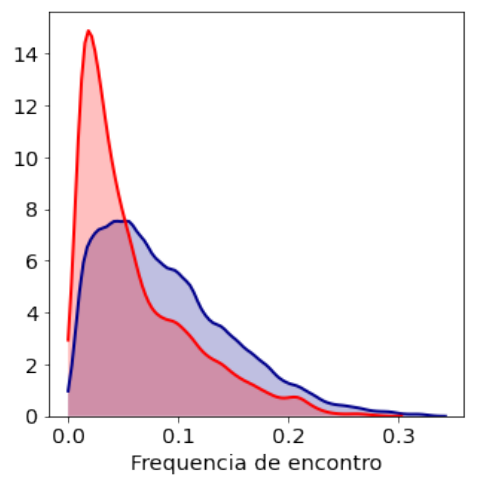

(a)

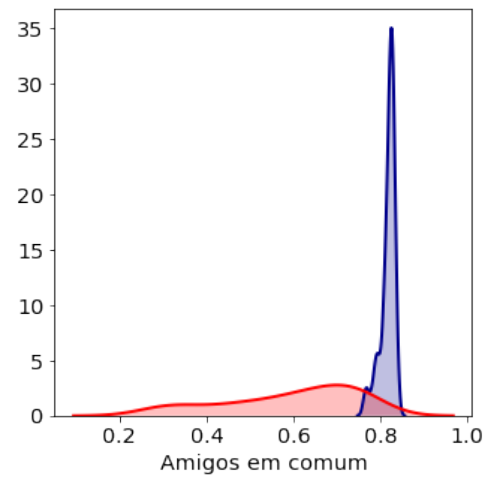

(b)

Figura 3. Densidade de probabilidade EDGEP (a), e TOPO (b) dos objetos fixos (vermelho) e móveis (azul)

\begin{tabular}{|c|c|c|}
\hline DISPOSITIVO & INCO & MAXCON \\
\hline Smartphone & 12580.9 & 28248 \\
\hline Laptop & 12867.4 & 20154 \\
\hline Tablet & 12823.6 & 11719 \\
\hline Smartwatch & 12324.1 & 3398 \\
\hline Smartband & 12564.1 & 2928 \\
\hline SmartTV & 15767.4 & 1609 \\
\hline Headset & 11916.2 & 1321 \\
\hline Games Console & 15082.8 & 1017 \\
\hline TV Streaming Device & 15241.9 & 528 \\
\hline Smart Home Product & 18266.7 & 440 \\
\hline
\end{tabular}

tra que o raio de giro dos objetos móveis é em média de 1300 unidades, porém o TRVD é em média 30. Isso implica que objetos tendem a fazer viagem menores com mais frequência, mesmo esporadicamente visitando pontos distantes. Isto se relaciona com o modelo de mobilidade utilizado (SWIM), além disso, conclusões similares foram apontadas por de Souza et al. [2018].

Por fim, a análise da entropia dos contatos, CONEN, reforça que objetos que se movem tendem a se encontrar com uma diversidade maior de dispositivos, assim como a entropia espacial, SPAV, em que os nós móveis tendem a visitar lugares diversos. É interessante salientar que a entropia é particularmente atraente porque os objetos de alta entropia, por definição, são precisamente aqueles com maior probabilidade de realizarem encontros não casuais. Ou seja, usuários de diferentes contextos têm maior probabilidade de se conectarem com dispositivos 'não-amigos'.

Com relação ao protocolos de comunicação, foram consideradas duas métricas tradicionais. A primeira é a taxa de entrega de mensagens que avalia a porcentagem de mensagens entregues com sucesso para diferentes valores de Time to Live (TTL) da mensagem. A segunda é o número de transmissões que pode ser interpretado como o custo ou 
sobrecarga de controle (overhead), em número de transmissões dispositivos-a-dispositivo, para entregar as mensagens para diferentes TTLs. Uma das metas dos algoritmos oportunísticos é ser custo-eficientes na entrega de mensagens visando apresentar a maior taxa de entrega com o menor overhead na rede.

A avaliação do desempenho desses protocolos de encaminhamento oportunístico é exibido na Figura 4. Na Figura 4(a) percebe-se que a estratégia Epidemic apresenta um limite superior para a entrega de mensagens e é a mais rápida em entregar as mensagens, isto condiz com sua abordagem de inundar a rede. Na sequência, o protocolo PRoPHET apresentar curva de entrega que o destaca em relação ao Fisrt Contact e Direct Delivery, isto acontece porque o PRoPHET possui uma "consciência" social e espacial para encaminhar mensagens de modo mais eficiente. First Contact e Direct Delivery apresentam desempenho de entrega similares, sendo que o First Contact entrega um pouco a mais de mensagens do que o Direct Delivery quando se tem um longo período.

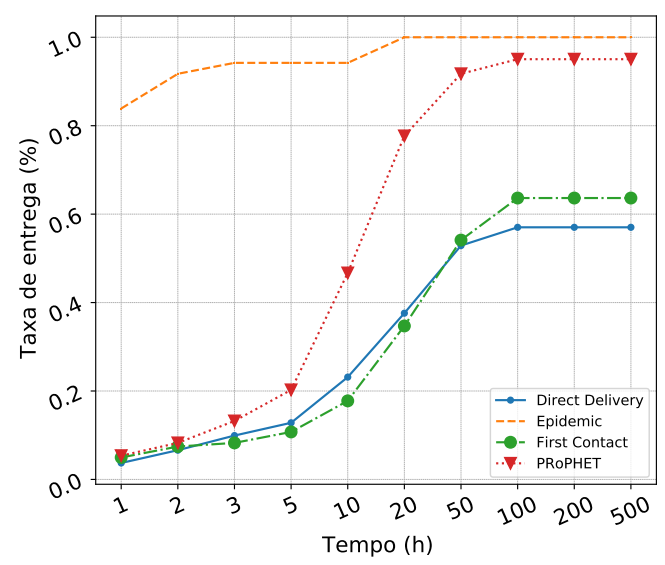

(a) Entrega de dados

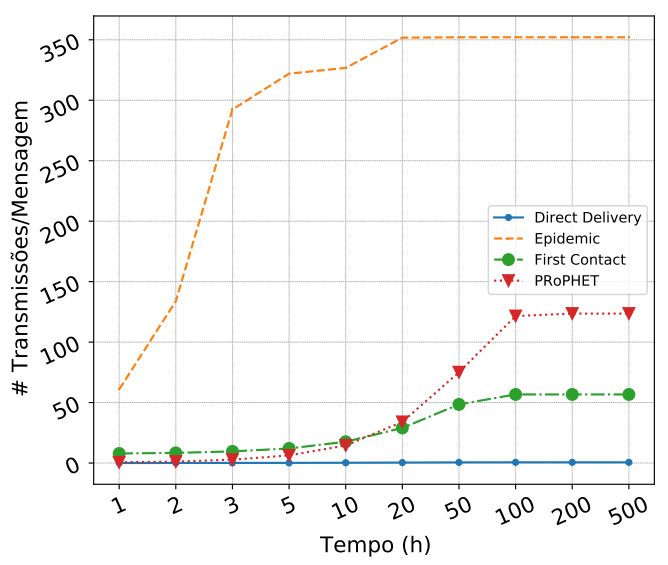

(b) Sobrecarga de controle

Figura 4. Desempenho de diferentes protocolos de encaminhamento oportunísticos em traces sintéticos de dispositivos.

A sobrecarga de controle para diferentes TTLs para os diferentes protocolos é apresentada na Figura 4(b), O protocolo Epidemic apresenta o maior custo e isto é esperado devido a inundação que faz. Isso mostra que o Epidemic não escala bem quando comparado com as demais estratégias. PRoPHET apresenta custo intermediário, o que pode ser interpretado aqui como uma boa estratégia custo-eficiência. Direct Delivery e Fisrt Contact apresentam o primeiro e o segundo melhores custos, porém não apresentam as melhores taxas de entrega de mensagens.

\section{Conclusão}

A IoT é uma realidade em nosso cotidiano, tal que objetos inteligentes estão cada vez mais onipresentes, seja nas nossas casas, nos nossos serviços ou em nossos bolsos. Por isso, entender como esses objetos se comportam em um contexto móvel abre portas para a construção de soluções eficientes, auxiliando na tomada de decisões em direção à ubiquidade dos elementos computacionais. 
O estudo aqui apresentado visou dar passos com o intuito de melhorar o entendimento sobre o comportamento social das "coisas" na Internet. Com esse intuito, foi manipulado um conjunto de dados considerando objetos móveis e estáticos, os quais foram disponibilizados online. Além disso, foram aplicadas uma série de métricas quantitativas e análises que consideraram não apenas as interações sociais dos dispositivos, como também suas características de mobilidade e comunicação. Com isso, no modelo ensaiado, foi possível ressaltar a relevância dos objetos móveis quando se trata do número de conexões realizadas e da diversidade desses contatos, sobretudo os smartphones que se destacam nas centralidades analisadas dentro da rede de objetos formada. Portanto, cabe inferir que devido à alta regularidade de encontros com dispositivos diversos e a capacidade de formar laços fortes dentro da comunidade, os objetos móveis desempenham papel chave em redes oportunísticas.

É importante frisar que pesquisas futuras devem ser realizadas para conjuntos de dados mais completos. É preciso avaliar os resultados das nossas análise com outros dados de mobilidade, tanto reais, como também utilizar outros modelos sintéticos trazendo novas perspectivas que aproximem ainda mais o modelo da realidade. Por fim, pretendese ainda propor novas métricas relacionadas a análises de dados no contexto da SIoT.

\section{Referências}

A. Aljubairy, W. E. Zhang, Q. Z. Sheng, and A. Alhazmi. Siotpredict: A framework for predicting relationships in the social internet of things. In International Conference on Advanced Information Systems Engineering, pages 101-116. Springer, 2020.

H. Z. Asl, A. Iera, L. Atzori, and G. Morabito. How often social objects meet each other? analysis of the properties of a social network of iot devices based on real data. In 2013 IEEE Global Communications Conference (GLOBECOM), pages 2804-2809, 2013. doi: 10.1109/GLOCOM.2013.6831499.

L. Atzori, A. Iera, G. Morabito, and M. Nitti. The social internet of things (siot)-when social networks meet the internet of things: Concept, architecture and network characterization. Computer networks, 56(16):3594-3608, 2012.

L. Atzori, C. Campolo, B. Da, R. Girau, A. Iera, G. Morabito, and S. Quattropani. Smart devices in the social loops: Criteria and algorithms for the creation of the social links. Future Generation Computer Systems, 97:327-339, 2019.

G. Bigwood, D. Rehunathan, M. Bateman, T. Henderson, and S. Bhatti. Exploiting selfreported social networks for routing in ubiquitous computing environments. In 2008 IEEE International Conference on Wireless and Mobile Computing, Networking and Communications, pages 484-489. IEEE, 2008.

BonnMotion. BonnMotion a mobility scenario generation and analysis too. Downloaded from http://sys.cs.uos.de/bonnmotion/index.shtml, abr 2016.

M. Castro, A. J. Jara, and A. F. Skarmeta. An analysis of $\mathrm{m} 2 \mathrm{~m}$ platforms: Challenges and opportunities for the internet of things. In 2012 Sixth International Conference on Innovative Mobile and Internet Services in Ubiquitous Computing, pages 757-762, 2012. doi: 10.1109/IMIS.2012.184.

S. Chang, E. Pierson, P. W. Koh, J. Gerardin, B. Redbird, D. Grusky, and J. Leskovec. Mobility network models of covid-19 explain inequities and inform reopening. Nature, 589(7840):82-87, 2021.

F. R. de Souza, A. C. Domingues, P. O. Vaz de Melo, and A. A. Loureiro. Mocha: A tool for mobility characterization. In Proceedings of the 21st ACM International Conference 
on Modeling, Analysis and Simulation of Wireless and Mobile Systems, pages 281-288, 2018.

N. Eagle, A. S. Pentland, and D. Lazer. Inferring friendship network structure by using mobile phone data. Proceedings of the national academy of sciences, 106(36):1527415278, 2009.

D. Hristova, M. J. Williams, M. Musolesi, P. Panzarasa, and C. Mascolo. Measuring urban social diversity using interconnected geo-social networks. 25th International World Wide Web Conference, WWW 2016, pages 21-30, 2016.

R. L. Inocêncio and F. N. Ribeiro. Inferring Cultural Similarity among Brazilian States Based on Data from Social Media Advertising Platforms, page 261-268. Association for Computing Machinery, New York, NY, USA, 2020. ISBN 9781450381963.

W. Kassab and K. A. Darabkh. A-Z survey of internet of things: Architectures, protocols, applications, recent advances, future directions and recommendations. Journal of Network and Computer Applications, 163:102663, 2020.

A. Keränen, J. Ott, and T. Kärkkäinen. The one simulator for dtn protocol evaluation. In Proceedings of the 2nd international conference on simulation tools and techniques, pages 1-10, 2009.

V. Kostakos and J. Venkatanathan. Making friends in life and online: Equivalence, microcorrelation and value in spatial and transpatial social networks. In 2010 IEEE Second International Conference on Social Computing, pages 587-594. IEEE, 2010.

A. Lindgren, A. Doria, and O. Schelén. Probabilistic routing in intermittently connected networks. ACM SIGMOBILE mobile computing and communications review, 7(3):1920, 2003.

C. Marche, L. Atzori, and M. Nitti. A dataset for performance analysis of the social internet of things. In 2018 IEEE 29th Annual International Symposium on Personal, Indoor and Mobile Radio Communications (PIMRC), pages 1-5. IEEE, 2018.

C. Marche, L. Atzori, V. Pilloni, and M. Nitti. How to exploit the social internet of things: Query generation model and device profiles' dataset. Computer Networks, 174:107248, 2020.

A. Mei and J. Stefa. Swim: A simple model to generate small mobile worlds. In IEEE INFOCOM 2009, pages 2106-2113. IEEE, 2009.

P. Meroni, S. Gaito, E. Pagani, and G. P. Rossi. CRAWDAD dataset the unimi/pmtr. Downloaded from http://www.crawdad.org/unimi/pmtr, sep 2010.

A. M. Meslin, N. d. L. R. Rodriguez, and M. Endler. Supporting multiple smart-city applications based on musanet, a common iomt middleware. In Anais do XVIII Workshop em Clouds e Aplicações, pages 13-26. SBC, 2020.

B. P. Santos, L. A. Silva, C. Celes, J. B. Borges, B. S. P. Neto, M. A. M. Vieira, L. F. M. Vieira, O. N. Goussevskaia, and A. Loureiro. Internet das coisas: da teoria à prática. Minicursos SBRC-Simpósio Brasileiro de Redes de Computadores e Sistemas Distribuidos, 31, 2016.

B. P. Santos, L. F. M. Vieira, and A. A. F. Loureiro. Routing and mobility management in the internet of things. In Anais Estendidos do XXXVIII Simpósio Brasileiro de Redes de Computadores e Sistemas Distribuídos, pages 161-168. SBC, 2020.

J. Surowiecki. The Wisdom of Crowds. Anchor, 2005. ISBN 0385721706.

J. M. Tjensvold. Comparison of the ieee 802.11, 802.15. 1, 802.15. 4 and 802.15. 6 wireless standards. In IEEE: September, volume 18, 2007.

D. Zinoviev. Complex network analysis in Python: Recognize-construct-visualizeanalyze-interpret. Pragmatic Bookshelf, 2018. ISBN 9781680502695. 